\title{
APPROACH TO CRITICAL CRACK OPENING DISPLACEMENT MODELING OF DAMAGE IN METAL SHEETS AFTER COMPOSITE PATCH BONDED REPAIR
}

\author{
Michal Salacinski ${ }^{1}$ \\ Andrzej Leski ${ }^{2}$ \\ Michał Stefaniuk ${ }^{1}$ \\ ${ }^{1}$ Air Force Institute of Technology, Ksiecia Boleslawa 6 street, 01-494 Warsaw, Poland \\ ${ }^{2}$ Military University of Technology, Faculty of Mechanical Engineering \\ michal.salacinski@itwl.pl, andrzej.leski@wat.edu.pl, michal.stefaniuk@itwl.pl
}

\begin{abstract}
The paper proposes a method of calculating the maximum displacement in the aircraft metal structure repaired by CPBR (bonded composite patch repair).

The calculations were made based on specimens. The specimens were prepared according to the current requirements used in aviation. The 2024-T3 alloy metal sheet was a structure. To repair the structure used the boron-epoxy composite patch in prepreg technology was used.

The metal structure was modeled as an isotropic elastic body. The metal structure coated with the composite patch was modeled as an orthotropic structure. Based on this, the stress was determined in the metal structure.

The size opening displacement in the metal structure was determined based on the model of linear elastic fracture mechanics for the plane stress state.

The calculation results were verified by measuring the displacement in laboratory conditions.

The laboratory tests made it possible to demonstrate the accuracy of the proposed approach.
\end{abstract}

Keywords: composites, aerospace, composite patch bonded repair.

\section{INTRODUCTION}

The use of polymer composites in repairing metallic structures is critical for maintenance of ageing aircraft. The composite patch repair method can be successfully applied to restore the technical condition of aircraft in which damage such as cracks and corrosion has been detected. Today, the composite and adhesives technology is highly developed and because of that it is widely used for repairing aircraft.

Advanced software analytical tools (including the Finite Element packages) provide information about the static strength and fatigue life of the structure. However, an initial parametric design is still needed as a starting point for the analysis (substantiation of repair). Basic calculations are needed to estimate the structural behavior after repair.

The present paper shows one approach to repair substantiation. A basic linear-elastic fracture mechanics and orthotropic model of the composite repaired metallic element are used to determine the relation rack opening as a function of applied load.

Design and manufacturing of notched specimens with composite repair patches of varying thickness is the first step of the research. Next, an analytical model is developed for establishing the relation between the crack opening and the load, for the particular specimen type. This step 
was supported by material tests. As a summary, the crack opening diagrams obtained are presented.

\section{SPECIMEN DESIGN}

Based on the assumption of the tensile character of the material tests, the designed specimens have two regions: the test region and the gripping region. The 2024-T3 aluminum alloy is used as the specimen material.
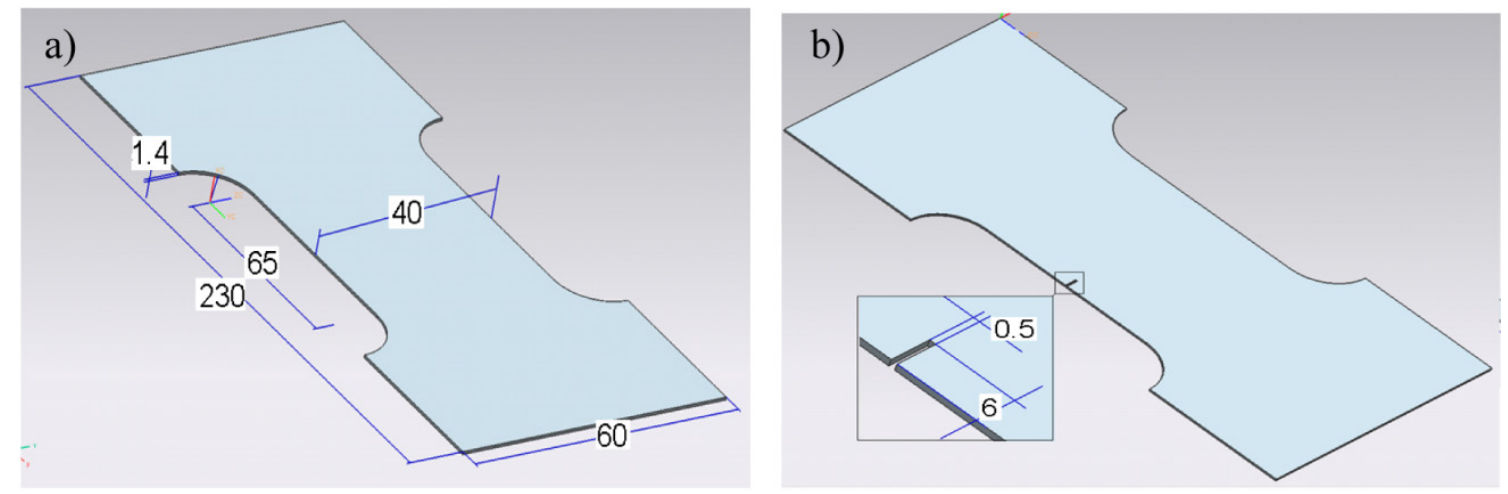

Fig. 1. The geometry of the sample; dimensions are in millimeters: a) blank sample - no damage modeled, b) sample with modeled damage without patches

To ensure the universality of the computational model, several configurations of the composite patch were used for the validation tests. Carbon-epoxy composite (CFRP - Carbon Fiber Reinfoced Plastics) was selected as the patch material. The number of lamina and their stacking sequence directly affects the strength and stiffness of the patch. As a whole, the system of the metallic structure with an applied patch can be classified as a hybrid, metallic-polymer composite CFRP and the 2024-T3.

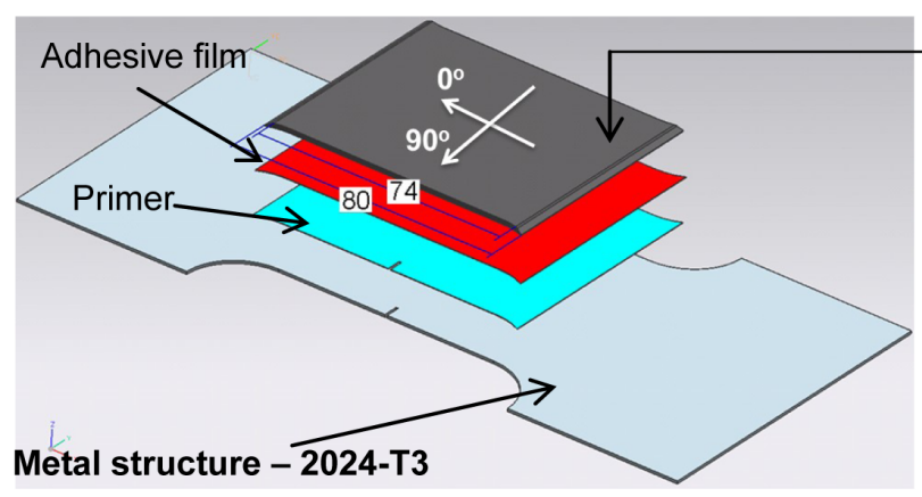

\section{Patch}

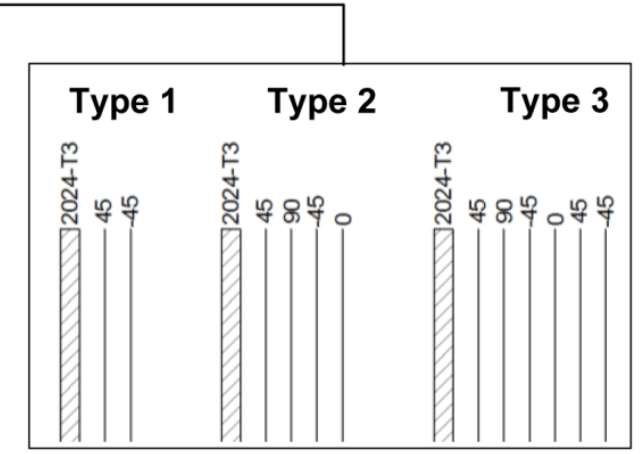

Fig. 2. The design of repair with patches: Type 1, Type 2, Type 3

\section{Specimen manufacturing}

The metallic specimens were made from a $1.4 \mathrm{~mm}$ thick 2024-T3 aluminum sheet, with the use of machining. The notch that models the crack was created with the use of a coping saw.

For the repair patch, aerospace-grade materials were used - unidirectional composite tape in the form of epoxy prepreg, and the epoxy adhesive film. Mechanical treatment was applied to the specimen surfaces, which were subsequently covered with sol-gel and a primer layer. Hardening was applied in a single heat cycle at a maximum temperature of $125^{\circ} \mathrm{C}$. 
a)

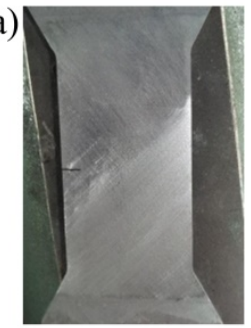

b)

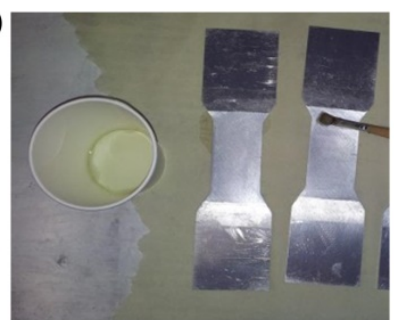

c)

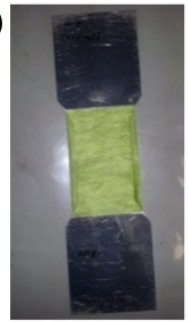

f)

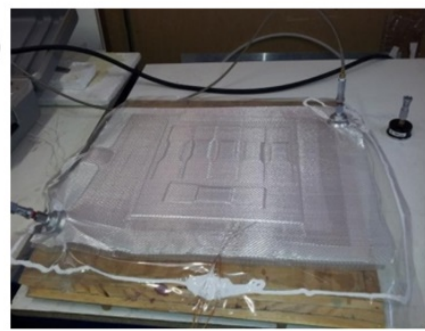

d)

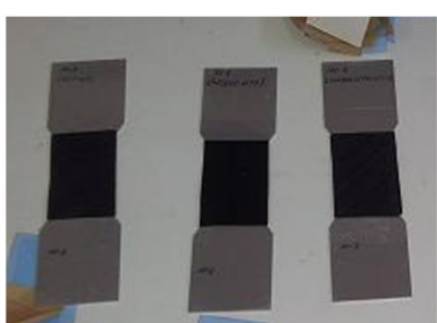

g)

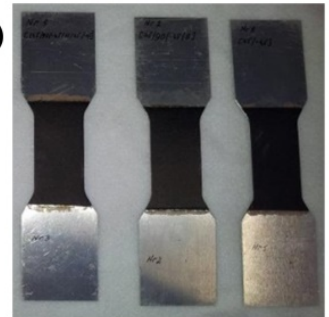

Fig. 3. Manufacturing process: a) mechanical treatment b)chemical treatment c) application of adhesive d)epoxy prepreg application e) heat cycle control unit f) vacuum bag g)completed specimens

\section{Modeling of the repaired metallic structure}

The metal-polymer hybrid can be treated as a laminar composite. This type of composite is modelled as an orthotropic structure subjected to plane stress (Fig. 5). Resultant forces and moments for the composites (according to figure 4) can be presented in the form of the matrix system of equations [1]:

$$
\left\{\begin{array}{l}
N \\
M
\end{array}\right\}=\left[\begin{array}{ll}
A & B \\
B & D
\end{array}\right] \cdot\left\{\begin{array}{l}
\varepsilon^{0} \\
\kappa^{0}
\end{array}\right\}
$$

$\mathrm{N}-1 \times 3$ Force vector

$\mathrm{M}-1 \times 3$ Bending Moment vector

$\varepsilon^{0}-$ Strain

$\kappa^{0}$ - curvature

A - (7) membrane Bendig stiffness matrix

$\mathrm{B}-$ (7) Coupling matrix, result of non-symmetry of the composite

D - plate stiffness matrix (7)

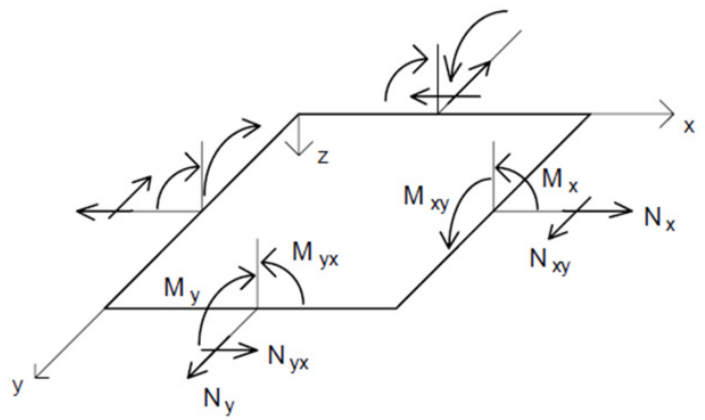

Fig. 4. Forces and bending moments in the plane of the composite [1]

Assuming that only tensile loads will be present, the system (1) can be reduced to the system below (2):

$$
N=\left[\begin{array}{ll}
A & B
\end{array}\right] \cdot \varepsilon^{0}
$$


After the transformations, an equation system (3) giving the stresses in each lamina is obtained, according to figure 5 :

$$
\sigma_{k}=\left[\overline{Q_{k}} \cdot A^{\prime}+z \cdot \overline{Q_{k}} \cdot B^{\prime}\right] \cdot N
$$

$\sigma_{\mathrm{k}}-1 \times 3$ stress vector

$\overline{Q_{k}}-3 \times 3$ reduced stiffness matrix

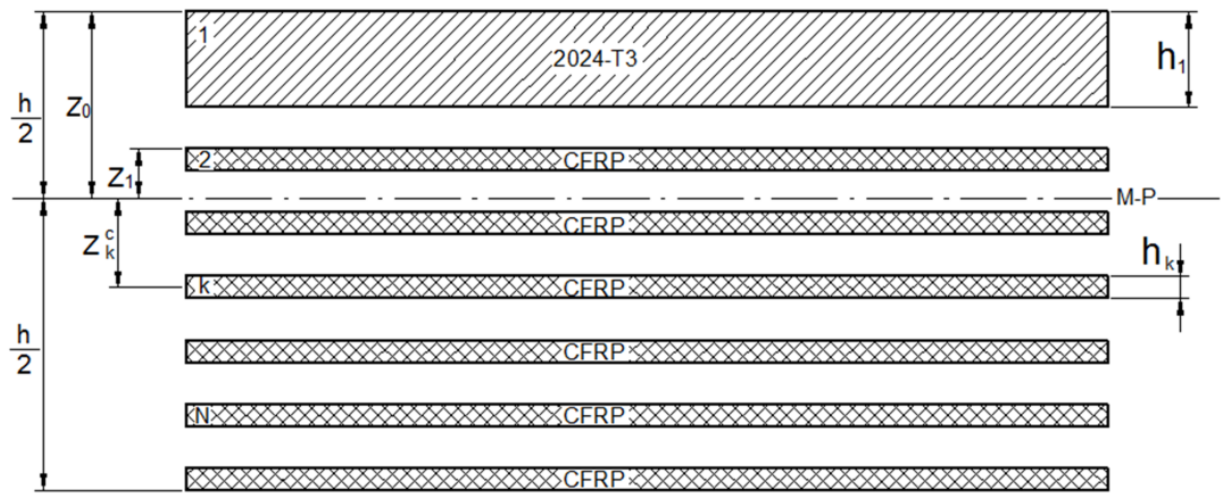

Fig. 5. Repair patch schematics - cross section of the metallic structure with the composite patch applied

\section{ENGINEERING CONSTANTS}

The input data for calculations were the engineering constants obtained from laboratory material tests. These are the values needed to describe material orthotopy. Based on those values the stiffnes matrix Q was obtained (Q) for a single unidirectional CFRP layer. The test load was applied in the direction parallel to the fibers.

$$
Q=\left[\begin{array}{ccc}
\frac{E_{x}}{1-v_{x} \cdot v_{y}} & \frac{E_{x} \cdot v_{y}}{1-v_{x} \cdot v_{y}} & 0 \\
\frac{E_{y} \cdot v_{x}}{1-v_{x} \cdot v_{y}} & \frac{E_{y}}{1-v_{x} \cdot v_{y}} & 0 \\
0 & 0 & G_{x y}
\end{array}\right]
$$

$E_{x}, E_{x y}$ - elastic moduli for principal material directions,

$v_{x}, v_{y}$ - Poisson ratio for principal material directions

$G_{x y}$ - shear module in the XY plane for a single layer

The reduced stiffness matrix $\overline{Q_{k}}$, describes the k-th composite layer rotated by the angle $\theta$.

$$
\overline{Q_{k}}=T^{-1} \cdot Q \cdot\left(T^{-1}\right)^{T}
$$

$T$ - transformation matrix

$$
T=\left[\begin{array}{ccc}
m^{2} & n^{2} & 2 \cdot m \cdot n \\
n^{2} & m^{2} & -2 m \cdot n \\
-m \cdot n & m \cdot n & m^{2}-n^{2}
\end{array}\right] \text {, where } m=\cos \theta, n=\sin \theta .
$$


When the reduced stiffnes matrix for each individual rotated lamina (including the metallic 2024-T3 layer) is known, the laminate stiffness matrices - A(membrane), B(coupling), $\mathrm{D}$ (plate) can be obtained (7):

$$
A=\sum_{k=1}^{N} \overline{Q_{k}} \cdot h_{k}, B=\sum_{k=1}^{N} \overline{Q_{k}} \cdot h_{k} \cdot z_{k}^{c}, D=\sum_{k=1}^{N} \overline{Q_{k}} \cdot\left(h k \cdot\left(z_{k}^{c}\right)^{2}+\frac{h_{k}^{3}}{12}\right)
$$

The following auxillary matrices were used to make the calculations easier:

$$
H=D-B \cdot A^{-1} \cdot B, A^{\prime}=A^{-1}+A^{-1} \cdot B \cdot H^{-1} \cdot B \cdot A^{-1}, B^{\prime}=-A^{-1} \cdot B \cdot H^{1}
$$

According to the formula (3), the metallic structure stresses $\sigma_{k}(\mathrm{k}=1)$ can be obtained. Therefore:

$$
\sigma_{1}=\sigma_{2024}=\left\{\begin{array}{l}
\sigma_{2024}^{1} \\
\sigma_{2024}^{2} \\
\tau_{2024}
\end{array}\right\}
$$

Stresses remote to the crack equal.

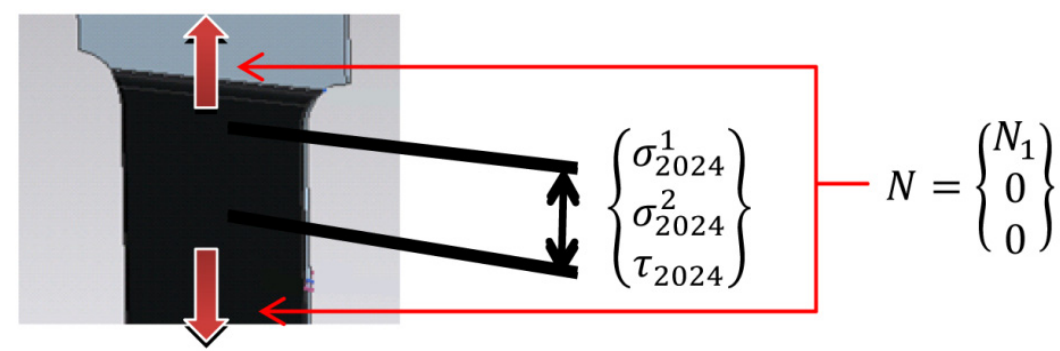

Fig. 6. Calculated stresses under the composite patch

\section{Calculation of the crack opening}

Based on the linear elastic fracture mechanics for plane stress [2], the crack opening displacement as a function of remote stresses and crack length can be obtained:

$$
u_{1}=\frac{2}{E_{2024}} \cdot \sigma^{\infty} \cdot a
$$

$a$ - crack lenght,

$\sigma^{\infty}-$ remote stress.

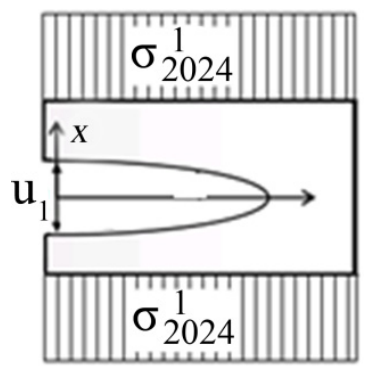

Fig. 7. Crack geometry and applied loads 
After obtaining stress values (9) and substituting $\sigma_{2024}^{1}$ into the equation (10), the crack opening displacement under the composite patch is calculated (Fig. 7):

$$
u_{1}=\frac{2}{E_{2024}} \cdot \sigma_{2024}^{1} \cdot a
$$

\section{TESTS OF SPECIMENS WITH REPAIR}

Testing was performed at the AFIT material testing laboratory. The static strength tests were done with the extension velocity of $0.8 \mathrm{~mm} / \mathrm{min}$, and a $50 \mathrm{kN}$-grade force sensor was used. During the test, the tensile force and the crack extension (obtained via an videoextensometer) were recorded.
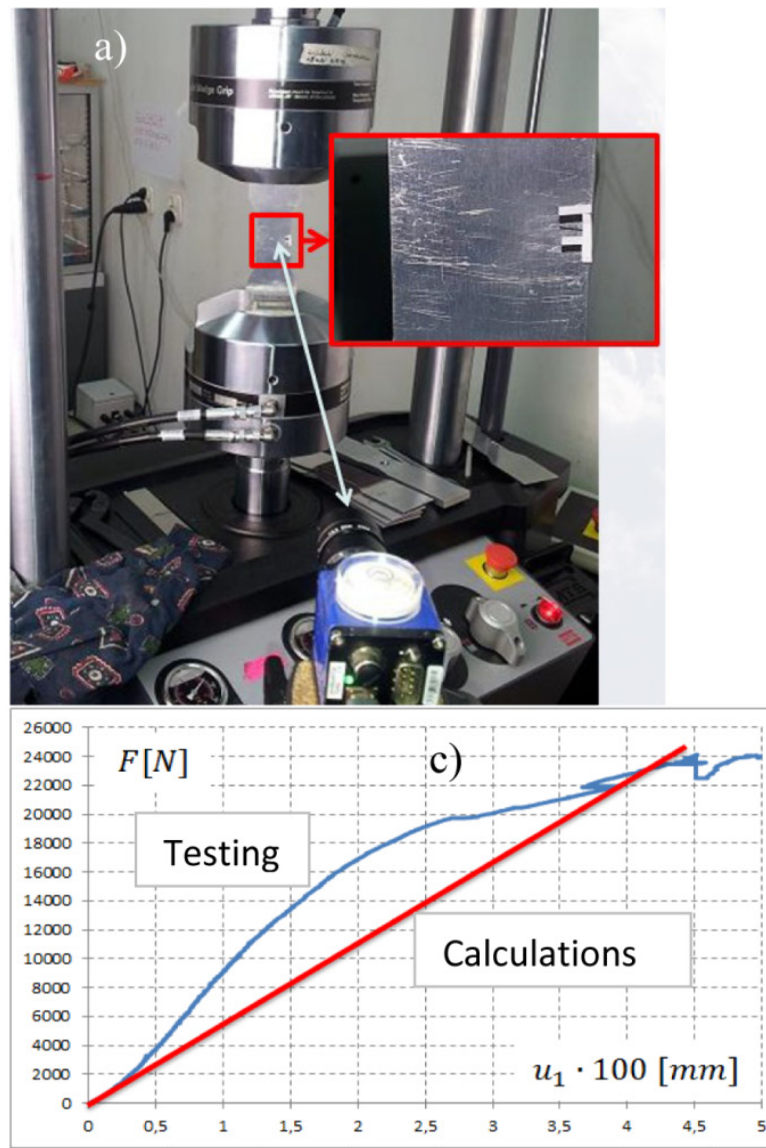
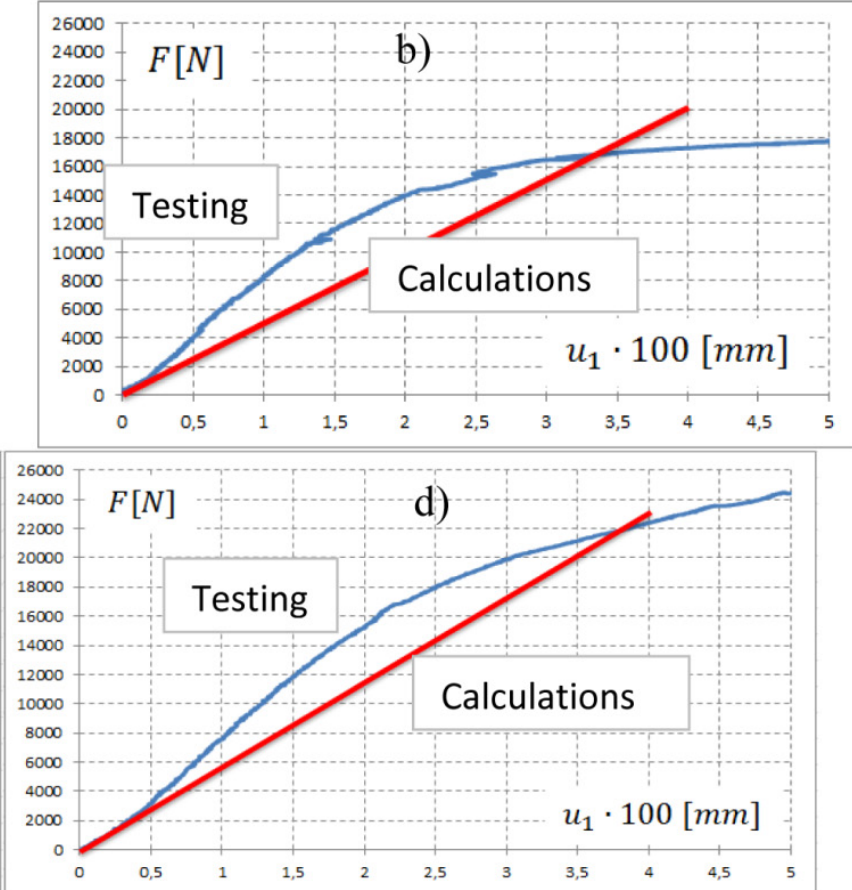

Fig. 8. Diagrams comparing the model calculations and mean material test values for the notched specimens after repair: a) test stand with a specimen and a videoextensometer installed, b) repair patch [45/-45] applied, c) repair patch [45/90/-45/0] applied, d) repair patch [45/90/-45/0/45/-45] applied

\section{CONCLUSIONS}

Testing was conducted for three sets of specimens with different configurations of laminae in the composite patch. Each set consisted of three specimens. Based on the averaged test results comparative diagrams were created (Fig. 8). Mean standard deviations for all of the specimens equaled $\mathrm{S}_{\mathrm{F}}=324 \mathrm{~N}$ for the force value and $\mathrm{S}_{\mathrm{u} 1}=0,00002 \mathrm{~mm}$ for the COD value. 


$$
\eta_{i}=\frac{u_{c a l}-u_{\text {meas }}}{u_{c a l}} \cdot 100 \%
$$

$u_{c a l}-$ calculated COD for the point of highest discrepancy between test and calculation,

$u_{\text {meas }}-$ measured COD for the point of highest discrepancy between test and calculation,

$\eta_{i}$ - deviation resulting from differences in patch configuration $(i-$ specimen number - Fig. 2$)$

The average difference between test and calculation with the force held constant (formula (12)) equals $\eta_{1}=32 \%, \eta_{2}=31 \%, \eta_{3}=28 \%$. Altough the difference is quite significant, its value is repeatable for a given patch configuration. The authors consider the possibility to introduce a correction coefficient for the mathematical model. This requires performing tests for a larger set of cases, which will be done in the further course of research.

\section{REFERENCES}

[1] J. German, (2001). Podstawy mechaniki kompozytów włóknistych, Politechnika Krakowska, Kraków.

[2] T. L. Anderson, (1995). Fracture Mechanics: Fundamentals and Applications. CRC Press. 